\title{
Superficial Myofibroblastoma: Unusual Hymenal Localization
}

\author{
K Harou $^{1,3}$, J Ouchraa ${ }^{1,3^{*}}$, S Houari ${ }^{1,3}$, A Aboulfalah ${ }^{1,3}$, H Rais ${ }^{2,3}$, A. Soummani ${ }^{1,3}$ \\ ${ }^{1}$ Gynecology-obstetrics Department, University Hospital Mohammed VI Marrakech \\ ${ }^{2}$ Department of Anatomic Pathology, University Hospital Mohammed VI, Marrakech \\ ${ }^{3}$ Cadi Ayyad University, Marrakech, Morocco
}

DOI: $10.36348 /$ sijog.2020.v03i02.001

| Received: 29.01.2020 | Accepted: 06.02.2020 | Published: 09.02.2020

*Corresponding author: Ouchraa Jihane

Abstract

Superficial myofibroblastoma (SMF) of the lower female genital tract is a rare, recently recognized benign mesenchymal tumor. We report a very rare case of a superficial myofibroblastoma located in the hymenal ring of a 35 years old Virgin women. On examination, she had a circumscribed nodular mass, located in the posterior portion of the hymen, and exteriorized by the vulvar cleft, measuring approximately $3 \times 4 \mathrm{~cm}$. The surgical treatment was based on local resection, and an hymenoplasty to preserve the virginity of our patient. The histopathological examination revealed a hypocellular tumor, composed of spindle tumor cells with myofibroblastic differentiation, and the presence of nuclear polymorphism, making it difficult to distinguich from a sarcamatous origin. The immunohistochemistry evaluation helped to obtain the diagnosis of SMF, by showing reactivity for estrogen and progesterone receptors, desmin, CD34. The knowledge of the clinicopathological aspect of superficial myofibroblastma is essential to distinguish it from the other mesenchymal lesions of the lower female genital tract. The treatment of this particular localization should consider the preservation of the hymenal ring especially in our cultural context.

Keywords: Myofibroblastoma, hymenal ring, hymenoplasty.

Copyright @ 2020: This is an open-access article distributed under the terms of the Creative Commons Attribution license which permits unrestricted use, distribution, and reproduction in any medium for non-commercial use (NonCommercial, or CC-BY-NC) provided the original author and source are credited.

\section{INTRODUCTION}

Superficial myofibroblastoma is a very rare, benign mesenchymal tumor of the female genital region. Recently named by Ganesan R et al. In 2005, as superficial myofibroblastoma of the lower female genital tract, because it occurs in the cervicovaginal area, and also in the vulva [1]. To the best of our knowledge, only 46 cases have been reported in the English language literature, and only 7 cases have been reported to arise in the vulva $[1,2,5]$. Histologically, this benign tumor is characterized by myofibroblast proliferation in collagenous and myxoid stroma. It may be associated with extensive edema in the stroma, which makes it difficult to distinguish from aggressive tumors of this area [1-3]. In the present report, we describe an original case of an hymenal localization of superficial myofibroblastoma, occurring to a 35 years old virgin woman. This localization of superficial myofibroblastoma on the hymenal ring is probably the first one to be reported in English literature so far. Our report aims to describe this original localization, and to highlight diagnosis difficulties and the management dilemma of virginity sparing surgery.

\section{CASE REPORT}

We report the case of a 35 years old woman, nulligest and nulliparous, virgin, presented with a tumor in her hymen, increasing in size progressively. She never had any hormonal therapy in the past, and she denied any significant current or past medical conditions. There were no abnormalities detected in the general and systematic examination. The gynecologic exam revealed a well circumscribed nodular mass. The tumor was located in the posterior portion of the hymenal ring, it was exteriorized by the vulvar cleft, measuring approximately $3 \times 4 \mathrm{~cm}$ (figure $1(\mathrm{~A})$ ). No additional gynecological pathologies were observed based on patient examination and transvaginal ultrasonography. Magnetic resonance imaging (MRI) of the pelvis showed a well-circumscribed $6,5 \mathrm{~cm}$ mass in the posterior wall of the vulva (figure 2). The lesion was excised to include normal tissue margins, and was 
sent to the pathology department for evaluation. Respecting the desire of our patient, a virginity sparing surgery including hymenal reconstruction was performed (figure1 $(\mathrm{B}, \mathrm{C})$ ). The result after 3 months provides total satisfaction to the patient (figure1 (D)).

Gross examination of the mass showed a 4 , $5 \times 3,5 \times 2.5 \mathrm{~cm}$ ovular mass, soft to firm, without any necrosis or induration, the cut surface was whitish, the boundary between tumor and adjacent tissue was clear. Microscopic examination revealed a wellcircumscribed, unencapsulated tumor, the stroma was edematous and myxoid, with the presence of short and spindle shaped cells with nuclear pleomorphism and hyperchromatism, suggesting initially a pseudo sarcomatous origin (figure 3 ).

On immunohistochemical analysis, most tumor cells showed positivity for vimentin, Estrogen Receptor, Progesterone Receptor, and Desmin. Some tumor cells showed positive for CD31 and CD34. The Ki-67 labeling index was less than 5\%. This immunoprofile allowed us to establish the diagnosis of superficial myofibroblastoma (figure 4).

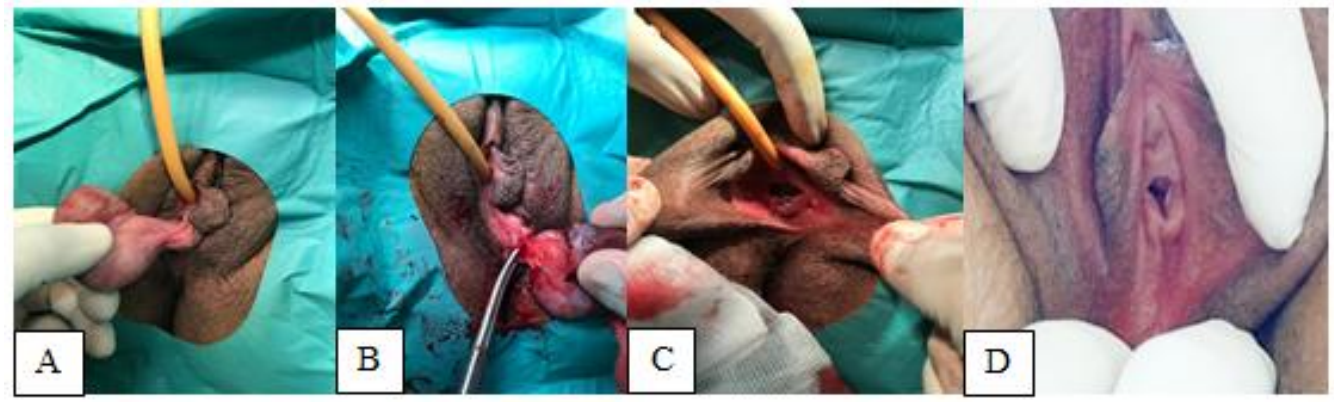

Fig-1: Superficial myofibroblastoma located in the hymenal ring

A : Nodular mass located in the posterior portion of the hymenal ring, exteriorized to the vulvar cleft

B : Surgical complete excision of the mass

C : Hymen reconstruction surgery

D : 3 months post-operative evolution

\section{DISCUSSION}

Superficial myofibroblastoma (SMF) of the lower female genital tract is a rare, benign mesenchymal tumor composed of spindle tumor cells with myofibroblastic like differentiation, Which characteristically arises from the subepithelial stroma of the vagina and, less frequently, of the vulva and cervix [4,5]. Clinically, SMF are described as a polypoid or nodular mass, the tumor size ranges from 2 to $65 \mathrm{~mm}$. it occurs to women aged between 23 and 80 years old [4, 5]. The pathogenesis remains unclear, but it could be hormone-related, because some number of cases had been administering tamoxifen, however no association with viral infection has been found [4,5]. The imaging modalities do not contribute much to the diagnosis of $\mathrm{SMF}$, it can appear in Magnetic resonance imaging (MRI) as a well-circumscribed mass in the vagina or the vulva $[2,6]$.

Diagnosing superficial myofibroblastoma is usually challenging because it is rare, and it shows many similar clinicopathologic features with the other genital mesenchymal tumors such as fibroepithelial stromal polyp, angiomyofibroblastoma, superficial angiomyxoma, inflammatory myofibroblastic tumor, and cellular angiofibroma $[1,2,4,5]$.

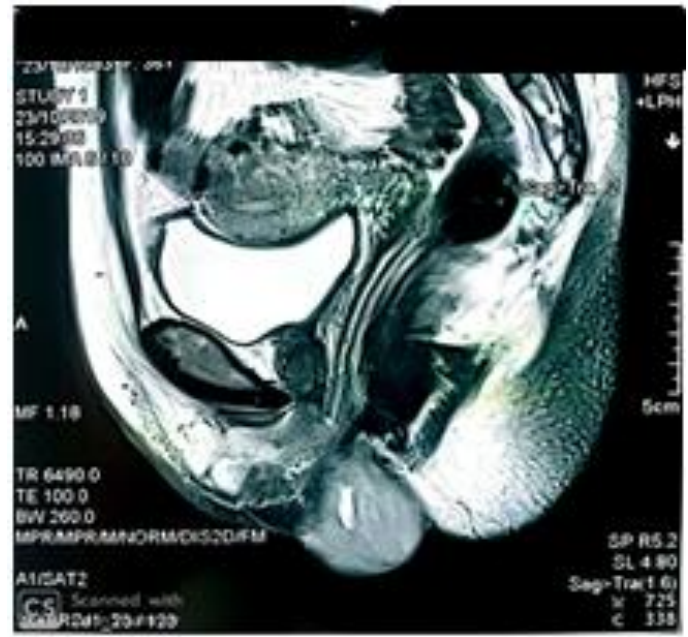

Fig-2: Magnetic resonance imaging of the pelvis showing a well circumscribed mass in the vulvar region.

Sometimes SMF histologically mimics aggressive angiomyxoma in which massive myxoid change in stroma is characteristic. The point of difference between the two of them is the location of the mass. SMF, as its name implies, presents as a mass in the superficial region, whereas Aggressive angiomyxoma is characterized by its deep location [2]. Moreover, the presence of spindle shaped cells, nuclear polymorphism, as well as prominent blood vessel proliferation in the stroma, made the present case atypical and difficult to distinguish from a sarcomatous origin. 


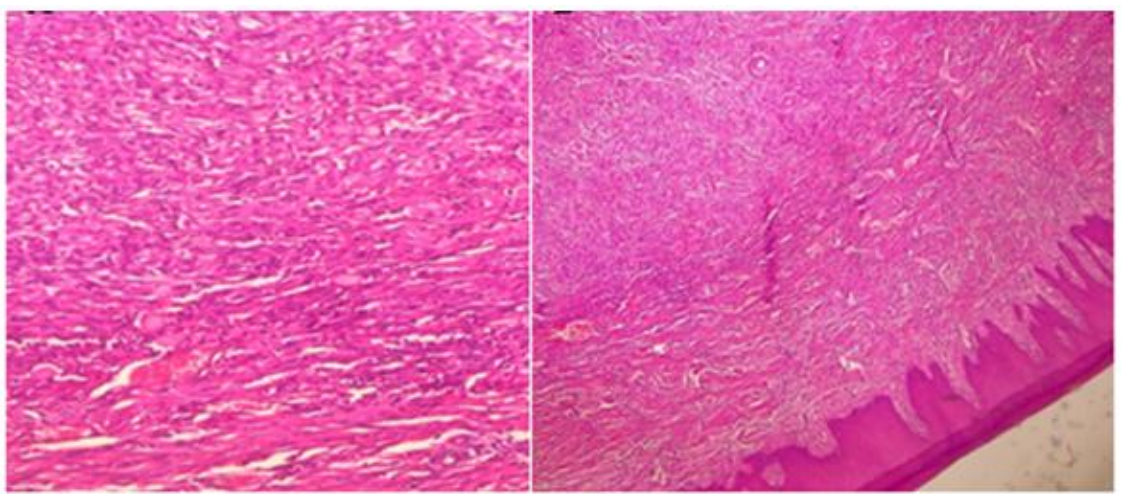

Fig-3 : Histological features of superficial myofibroblastoma showing spindle-shaped cells interrupted by collagen bands, with ovoid nucleus. The blood vessels are dilated and congestive.

The Immunohistochemistrical characterization is strongly suggested in leading to an accurate diagnosis, by showing positive for estrogen receptor, progesterone receptor, desmin, and some cases can also be reactive for smooth muscle actin (SMA) and CD34,
[2-4]. However, in some cases, the immunoprofile can be nonspecific and have many overlapping features with other mesenchymal tumors of the lower female genital tract $[1,2]$.

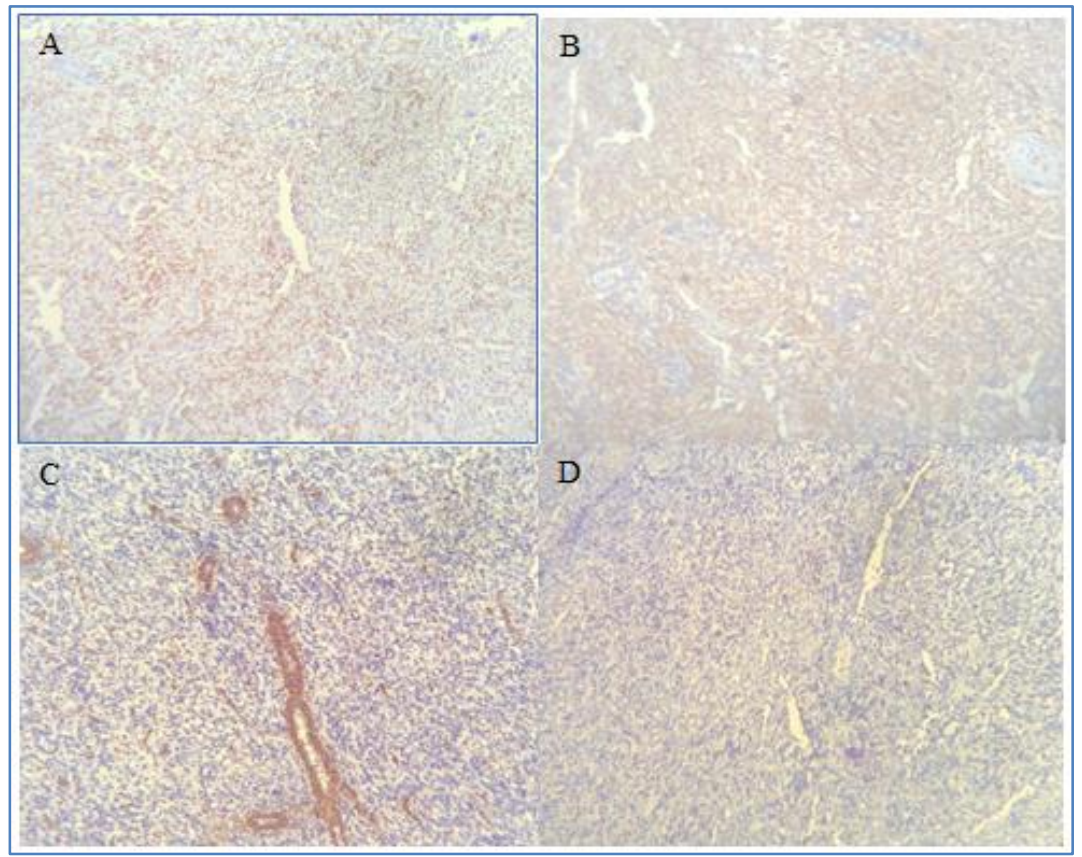

Fig-4: Immunohistological profile of Superficial myofibroblastoma showing positive nuclear staining for Estrogen Receptors (A). Tumor cells showed also cytoplastic positivity for CD34 (B), Smooth Muscle Actin (C), and Desmin (D)

The treatment of superficial myofibroblastoma of the lower female genital tract is based on a surgical resection of the tumor $[4,5]$. The challenge of this hymenal localization of superficial myofibroblastoma is to preserve the hymen in order to respect the patient desire to maintain her virginity, considering the cultural context.

The tumor has a benign course with no evidence of metastasis or recurrence with to exception of one vaginal case where local recurrence was developed 9 years after initial incomplete excision [4, 5].

\section{CONCLUSION}

The overall findings in our case are consistent with the rare entity of benign superficial myofibroblastoma. Awareness of the morphological and immunohistochemical heterogeneities of superficial myofibroblastoma of the lower female genital tract should facilitate its recognition and separation from other tumor-like lesions occurring in this area, mainly because of the different clinical prognoses. The therapeutic management of this particular localization on the hymenal ring can be a real dilemma because of the cultural considérations. 


\section{REFERENCES}

1. Ganesan, R., McCluggage, W. G., Hirschowitz, L., \& Rollason, T. P. (2005). Superficial myofibroblastoma of the lower female genital tract: report of a series including tumours with a vulval location. Histopathology, 46(2), 137-143.

2. Peng, W. X., Wada, R., Kure, S., Fukunaga, M., \& Naito, Z. (2019). Superficial Myofibroblastoma in the Vulva Mimicking Aggressive Angiomyxoma: A Case Report and Review of the Literature. Case reports in pathology, 2019.

3. Liu, J. L., Su, T. C., Shen, K. H., Lin, S. H., Wang, H. K., Hsu, J. C., \& Chen, C. J. (2012). Vaginal superficial myofibroblastoma: a rare mesenchymal tumor of the lower female genital tract and a study of its association with viral infection. Medical molecular morphology, 45(2), 110-114.
4. Abdelaziz, M., Eziba, N., Sharma, S., Kleven, D., \& Al-Hendy, A. (2017). Cervical superficial myofibroblastoma: Case report and review of the literature. SAGE open medical case reports, 5, $2050313 X 17726936$.

5. Magro, G., Caltabiano, R., Kacerovská, D., Vecchio, G. M., Kazakov, D., \& Michal, M. (2012). Vulvovaginal myofibroblastoma: expanding the morphological and immunohistochemical spectrum. A clinicopathologic study of 10 cases. Human pathology, 43(2), 243-253.

6. Atinga, A., El-Bahrawy, M., Stewart, V., \& Bharwani, N. (2019). Superficial myofibroblastoma of the genital tract: a case report of the imaging findings. BJR| case reports, 5(1), 20180057. 